\title{
Statistics for comparison of simulations and experiments of flow of blood cells *
}

\author{
K. Bachratá, H. Bachratý, M. Slavík
}

Cell-in-fluid research group, Department of Software Technologies, Faculty of Management Science and Informatics, University of Žilina, Slovakia, katarina.bachrata@fri.uniza.sk

\begin{abstract}
In this article we propose statistical method for comparison of simulation and real biological experiments of elastic objects moving in fluid. Our work is focused on future optimization of microfluidic devices used for capture of circulating tumor cells from blood samples. Since the design optimization using biological experiments is both time consuming and expensive, in silico experiments with a broad spectrum of complex and computationally simulations are intensely performed. Necessary verification if simulation models, hitherto mainly realised by comparision of individual cells properties must be extended to more complex simulations. We present our first results with characteristics designed for this purpose.
\end{abstract}

\section{Introduction}

Investigation of properties of elastic particles in the blood flow is a very broad field of research. Our research group focuses on the area of optimization of microfluidic devices intended to capture circulating tumor cells (CTCs) from blood samples. In this article, we use results of simulation experiments of blood flow through devices with system of internal obstacles and dimensions at the order of tens to hundreds of micrometers. For this purpose we have used

* All authors were supported by the Slovak Research and Development Agency [contract number APVV-15-0751]. a model implemented in an open source scientific package ESPResSo. The model consists of two parts: the fluid representing blood plasma and the immersed objects representing blood cells. The fluid is modeled using the latticeBoltzmann method and the objects are modeled by the immersed boundary methods implemented by our group. Objects are represented by triangular meshes of their surface and its elastic behavior is governed by five elastic moduli applied between nodes of the mesh. In this study we use only models of red blood cells (RBCs), which are the main component of the solid part of the blood suspen- 
sion and determine its behavior. More detailed description of our simulation model can be found in [1], [2], [7], [5]. Despite these specific conditions we believe that here presented results can be useful also for other types of experiments.

The main objective of simulations is to replace the biological experiments and bring results and knowledge about devices in cases where the biological experiments would be technologically too complex, too time-consuming or expensive. It is necessary to ensure and continuously maintain the accuracy and consistency of the simulation models and algorithms with the reality. This is accomplished mainly by comparing the simulation behavior to the biological experiments. Also in our research several (successful) comparisons were done, but almost all of them are related to the behavior and characteristics of the individual elastic objects in certain specific situations [9], [8], [4], [6]. Thanks to the research progress and to the increasing computational capability, it is also possible and necessary to verify the quality and precision of the simulation models in experiments with the flow of tens or hundreds of objects in larger devices. In addition to the comparison with biological experiments, there is also the possibility of comparing the results of identical in-silico experiments realised by different simulation tools and methods.

In both cases, our fundamental objective is to find methods, which gives a qualified answer to the question whether two experiments are consistent or not. According our main goals, we focus on results describing behavior and movement of microsized elastic particles in a steady, not turbulent flow. The main challenge is the big amount and rich variety of data needed to be collected from the simulation and then subsequently processed into a form, which allows to judge and measure the match of experiments. We have identified a relatively simple set of data for comparisons that can be obtained as output of simulation algorithms and also as results of image processing of visual records of biological experiments.

An example of the snapshot from real experiment with microfluidic devices is shown in the Figure 1.

Despite their simplicity, the data sufficiently describe the dynamics of the movement of tens or hundreds of objects in the experiment. They incorporate the stochastic features of experiment, the interactions between objects and device. It is clear, that direct comparison with a similar data set from another experiment is not possible. To enable the comparison, the data require a careful and sophisticated statistical processing including significant reduction into a suitable format. The aim of our paper is to present our first experience with designing, realization and implementation of such methods.

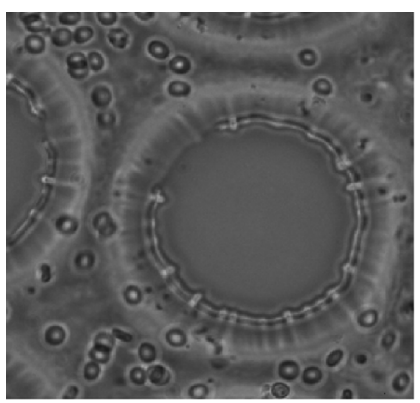

Fig. 1. Cells in microfluidic device, snapshot from the real experiment (personal communication with Dr. Giulia Mazza, Danube University Krems, July 2016). 


\section{Description of experiments and data}

Simulation experiments described in this work were performed in the microfluidic chamber in form of a box with a height of $30 \mu \mathrm{m}(z$-axis), width of $50 \mu \mathrm{m}(y$-axis) and a length of $100 \mu \mathrm{m}$ ( $x$-axis). The simulations included a cell-wall and fluid-wall interaction with top, bottom and sidewalls. To reduce the computational complexity, the simulation was periodic in the $x$ direction and its length could theoretically be unlimited. The cells in performed simulations used the lenght of channel up to $800 \mu \mathrm{m}$ in the $x$ direction. The assumption that the periodicity does not affect the results was also experimentally verified. Inside the microfluidic device, was placed a simple regular grid of cylindrical obstacles with diameter $10 \mu \mathrm{m}$ (Figure 2).

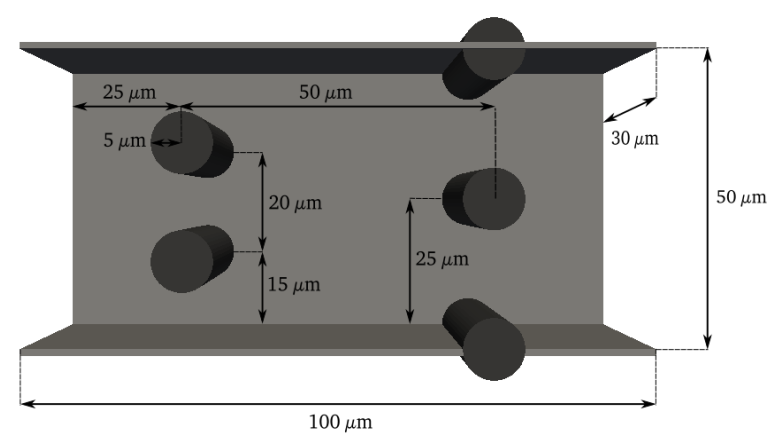

Fig. 2. Channel with five obstacles and its positions

Several different initial seedings were used to place 50 RBCs into the 'first period' of device (i.e. at the $x$-positions ranging from $0 \mu \mathrm{m}$ to $100 \mu \mathrm{m})$. Each cell was modeled using a triangulation of its surface with 141 nodes and identical elastic properties. In experiments $A$ and $B$ (Figure 3 ), the initial positions and skew of RBCs were generated randomly. To check the expected differences, in experiment
$C$ the cells were placed in regular grid positions and for experiment $D$ we have randomly generated 50 cells (as in $A, B)$, but with a modified channel topology where the central cylinder in the second row of obstacles was removed.

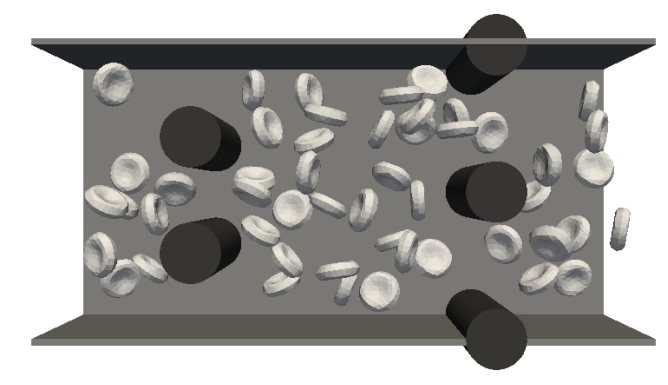

Fig. 3. Channel with five obstacles and randomly seeded cells for experiment $A$

The time step of simulation corresponds to $0.2 \mu$ s and according to 381000 simulation steps of each experiment the total simulated time was $0.0762 \mathrm{~s}$. The flow of blood plasma was initialized with fluid density 1 , fluid viscosity 1.5 and spatial step $1 \mu \mathrm{m}$. External volume force 0.0005 was used to induce the flow in the $x$ direction.

\subsection{Experiment snapshots}

We have obtained two sets of data from the simulations. The first set are 'simulation experiment snapshots'. Their meaning and content is motivated by the actual experiments, which are indeed often captured by one or more static images. For each snapshot at a fixed time we acquire the following information about each of the 50 RBCs: 
- minimum and maximum value of the $x, y, z$ - coordinates of cell surface (thus a cuboid containing the entire $\mathrm{RBC}$ and oriented in the direction of main the axes is defined)

- the position $(x, y, z$ coordinates $)$ of RBC center, defined as the center of circumscribed cuboid

The data are deliberately very simple. It should be troublefree get their counterparts from digital image processing of biological experiments. In this case, oftentimes $z$-coordinate will be missing. In the following, we will work with all three data dimensions, reducing them to only $x$ - and $y$ - dimension should not bring major problems.

\subsection{RBC movement tracking}

The second set of data is created by tracking the movement of all individual cells throughout the whole simulation. The aim was to continuously monitor particular characteristics of each RBC velocities in simulation. These data for in-silico experiment can be obtained with arbitrary precision with respect to the simulation algorithm step. For our needs it was sufficient to sample data every 200 simulation steps, which corresponds to time of $40 \mathrm{mi}-$ croseconds and $\mathrm{RBC}$ movement less than 0.5 micrometers. As the total length of the simulation was 381,000 steps, the movement data of each cell were tabulated at 1,909 points. For each RBC, we have obtained a sequence of the following data:
- The RBC center position $(x, y, z$ coordinates $)$ calculated as an average of coordinates of all surface triangulation nodes

$-x, y, z$ component of the RBC center velocity calculated as an average of the corresponding velocity components of all surface triangulation nodes

- absolute speed of the center calculated from $x, y, z$ speed components

- extremal points of RBC surface in terms of $x$-axis (the 'first' and 'last' surface point as seen the $x$ direction of the channel), $y$-axis ('most left' and 'most right' point) and $z$-axis ('top' and 'bottom' point)

$-x, y, z$ component of the velocity of the six extremal points of the RBC surface

We expect that these data should be available as outputs from other simulation tools as well. To obtain comparable data from biological experiments (with the possible loss of information about the $z$-coordinate), digitall processing of video experiment or a sufficiently detailed sequence of images must be performed. In any case, it is necessary to ensure the identification and tracking for each individual $\mathrm{RBC}$. In this case, it will be much easier to obtain the necessary data for comparison of in-silico experiments than for comparison of two in-silico to biological experiments.

\section{Cell-tracking data analysis}

\subsection{Absolute cell velocity}

As mentioned above, the values of velocity components along the $x, y$ and $z$ axes tabulated every 200 simulation 
steps were used for calculation of the absolute velocity for each RBC. This way we obtained a sufficiently precise vector of velocities for each of the 50 simulated cells.

The analysis of the individual velocity components indicates that the $x$ and $y$ directions are crucial, and neglecting the $z$-component would not cause a significant change of the following conclusions. This fact is important for comparing in-silico and biological experiments, where often only the top view is recorded and the $z$-axis coordinates information is lost.

The velocity plots (Figure 4) show a large diversity of the data and markedly different behavior of individual cells. To obtain the overall RBC movement characteristics, usage of statistical methods with further reduction of amount and relation of available data is needed.
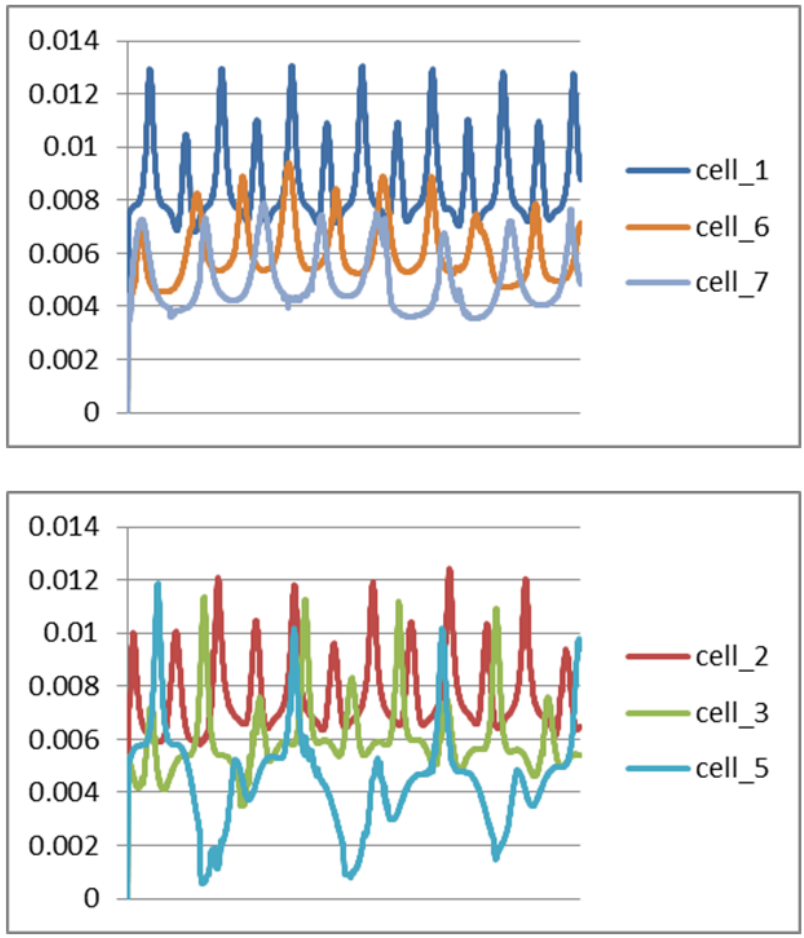

Fig. 4. Plot of speed graph for several RBCs

\subsection{Basic statistical analysis of the velocity data}

In the first step, we simply process the basic quantitative attributes of velocity vectors for each individual RBCs. For each velocity record we determined its minimum, maximum, range (i.e. the difference of maximum and minimum) and average of its values. (This average is not identical to the average value of the cell velocity, since the cell trajectory can be just extrapolate by linear curve dependent on the sampling frequency.)

Despite these simplification, the minimal value for each RBC captures the deceleration during a collision with an obstacle or when the cell is moving at the widest point of the channel. The maximum captures high velocity when the cells pass unconflictly the narrow parts of the channel. In range value the variability in cell movement and the overall ratio of 'slow' and 'fast' parts of its path is assessed.

Each of the monitored characteristics was stored in a separate vector and these were independently sorted by the magnitude of their components. These four vectors $\left(v \_\right.$min, $v \_$max $\left., v \_r n g, v \_a v g\right)$ represent the first overall characteristics of the movement of elastic objects. The comparison of their charts for simulations $A-D$ (Figure 5) shows the expected similarity and differences of the individual experiments. 

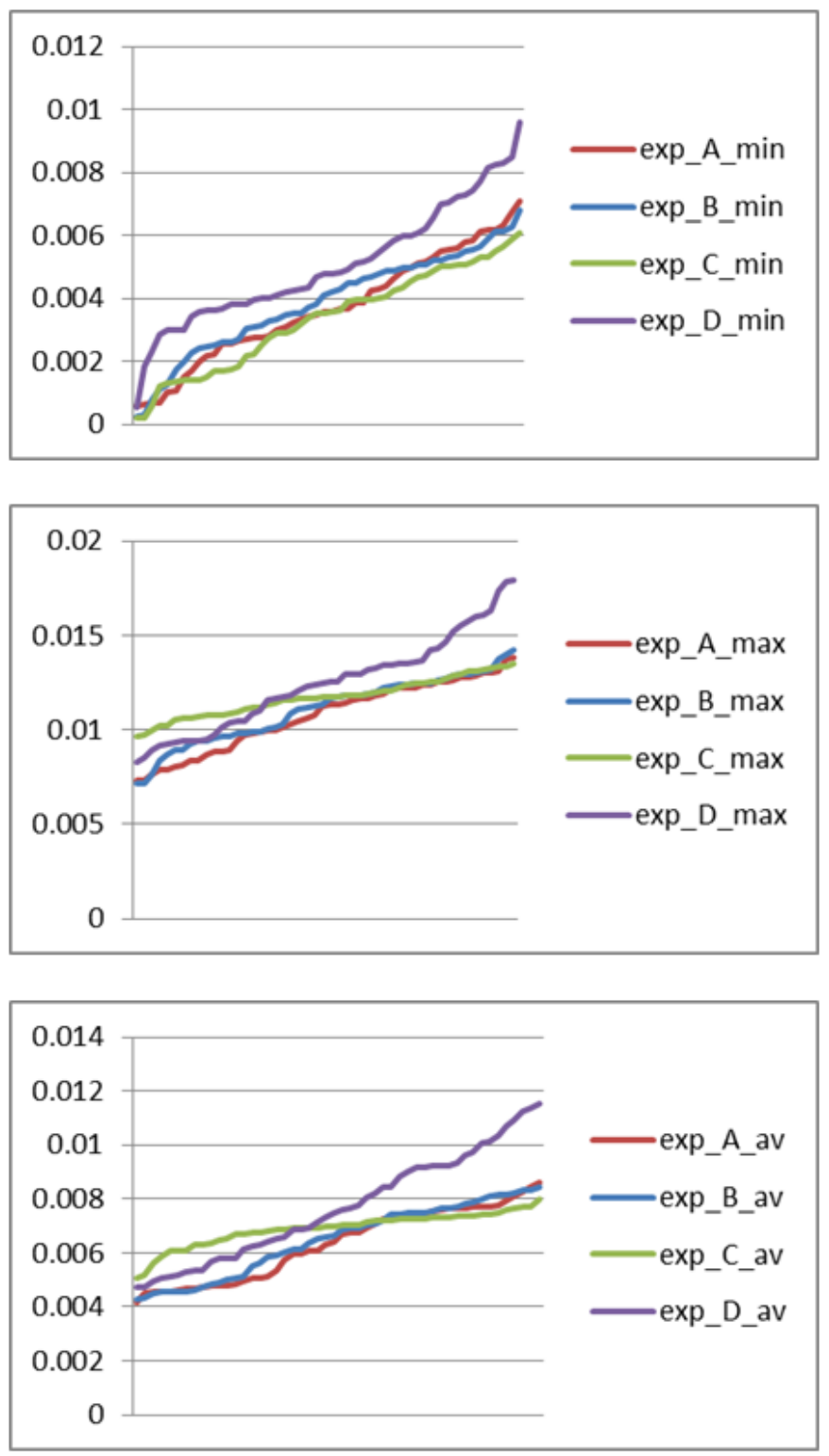

Fig. 5. Plot of vectors v_min, v_max and v_aver for simulations $A$ to $D)$.

We have quantitatively characterized this similarity using the standard Kolmogorov-Smirnov test (KS-test). Results for two of this characteristics are listed in the Table 1 and Table 2 . We have denoted as $h_{0}$ the statistic results, for which we do not reject the hypothesis $H_{0}$ that the measured data come from the same distribution, using the $\alpha=0.05$ significance level against alternative $H_{1}$. If we reject $H_{0}$, we denote it $h_{1}$.

Table 1. Kolmogorov Smirnov test for $v \_$max characteristic

\begin{tabular}{|c|c|c|c|c|}
\hline$v \_\max$ & $\mathbf{A}$ & $\mathbf{B}$ & $\mathbf{C}$ & $\mathbf{D}$ \\
\hline $\mathbf{A}$ & - & 0.6779 & 0.0044 & 0.0021 \\
\hline $\mathbf{B}$ & $h_{0}$ & - & 0.0089 & 0.0089 \\
\hline $\mathbf{C}$ & $h_{1}$ & $h_{1}$ & - & 0.0044 \\
\hline $\mathbf{D}$ & $h_{1}$ & $h_{1}$ & $h_{1}$ & - \\
\hline
\end{tabular}

Table 2. Kolmogorov Smirnov test for $v_{-} a v e r$ characteristic

\begin{tabular}{|c|c|c|c|c|}
\hline v_aver & $\mathbf{A}$ & $\mathbf{B}$ & $\mathbf{C}$ & $\mathbf{D}$ \\
\hline $\mathbf{A}$ & - & 0.9541 & 0.0021 & 0.0010 \\
\hline $\mathbf{B}$ & $h_{0}$ & - & 0.0021 & 0.0004 \\
\hline $\mathbf{C}$ & $h_{1}$ & $h_{1}$ & - & 0.0001 \\
\hline $\mathbf{D}$ & $h_{1}$ & $h_{1}$ & $h_{1}$ & - \\
\hline
\end{tabular}

\subsection{Analysis of the periodic characteristics of RBCs movement}

The velocity graphs clearly indicate that in addition to the extreme and average values, the periodicity of their behavior is another key feature. It can better characterize the topology of the channel, including the pattern of the obstacle array. We see an accelerated movement of cells in the narrow places and slower movement in wider parts of the channel. The topology is reflected in the trajectory of individual RBCs (how they slow down during collision with an obstacle, how they accelerate when passing obstacles without contact etc.). The irregularities in velocity 
periodicity correspond to collisions with other objects and their consequences. The number of periods also characterizes the overall velocity of the cell and its total covered distance.

The possibility to design our own criterions for periodicity of RBCs movement was very titilating, but we decided to use the Discrete Fourier Transform (DFT) as a standard method for periodic processes analysis. For each of the $50 \mathrm{RBCs}$ we have determined the three most important frequencies. For most of them, the first one was highly dominant.

This way we obtained a set of 50 dominant and $150 \mathrm{im}$ portant frequencies. The frecvencies of their values were sorted and saved into vectors $v_{-} f r e q \_d o m$ and $v_{-}$freq_imp. Thus we obtained a second much finer characteristic of the objects in flow velocities. The figures 6 and 7 show $v_{-}$freq_dom and $v_{-}$freq_imp and illustrate the degree of (dis)similarity of this characteristic for separate experiments. Even more significant results may be obtained when the frequencies are aggregated into groups of five (Figure 8). Comparison of experiments $A$ and $C$ shows that initial regular seeding of RBCs simplifies the frequency distribution. Finally, we have performed the KS test to find out whether the data come from the same distribution. This test has confirmed the results observed from graphs.
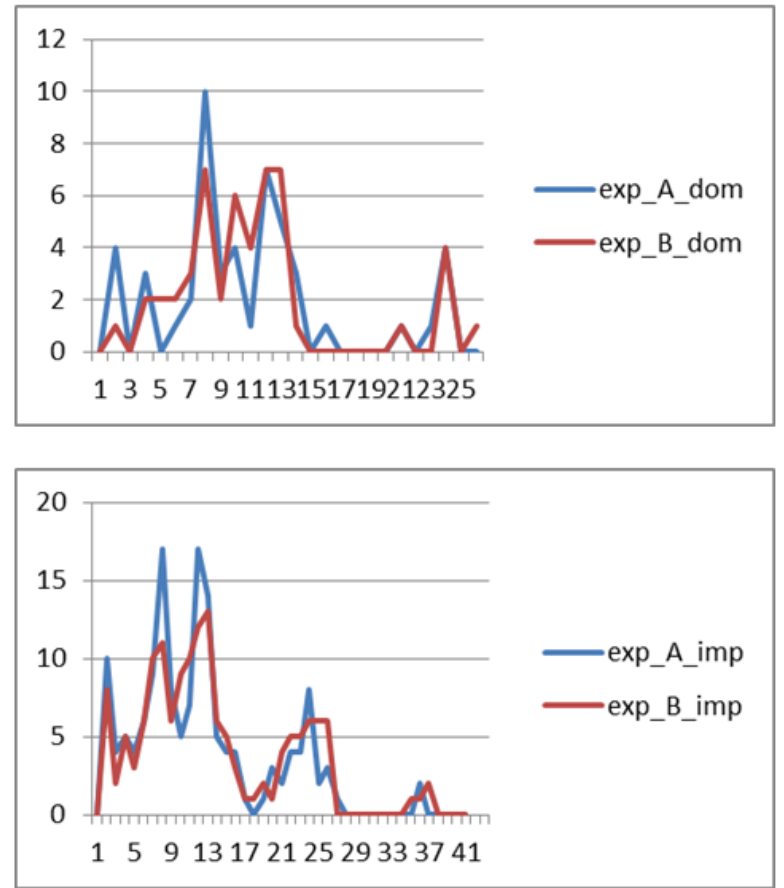

Fig. 6. Match in number of dominant and important frequencies for experiments $A$ and $B$
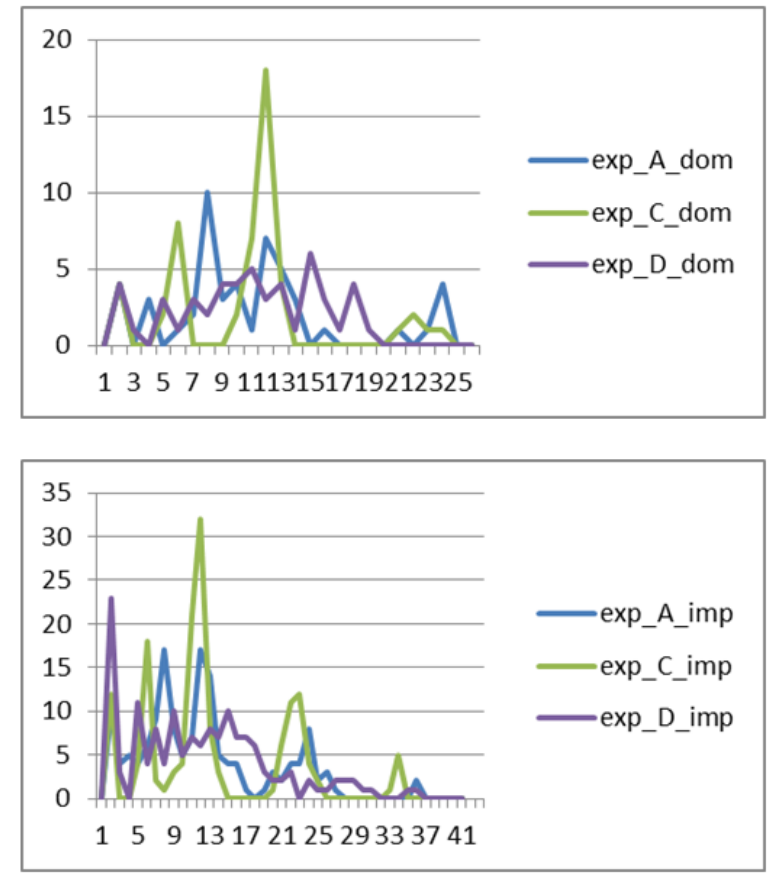

Fig. 7. Different course in number of dominant and important frequencies for experiments $A, C$ and $D$ 

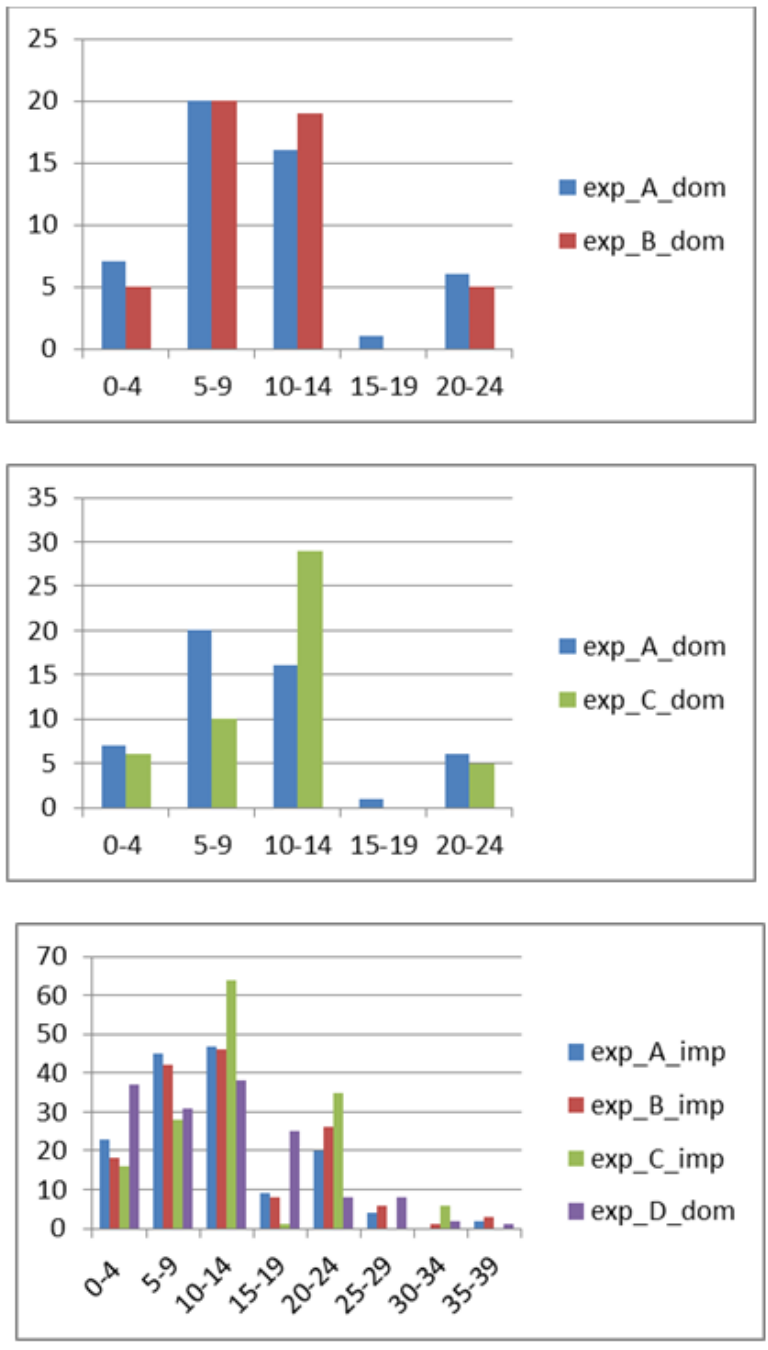

Fig. 8. Match of cumulated dominant frequencies for experiments $A$ and $B$, difference in cumulated dominant frequencies for experiments $A$ and $C$, numbers of cumulated important frequencies for all experiments

\subsection{Analysis of the cell rotation}

$\mathrm{RBC}$ rotation in the flow is another important characteristic of the experiment. It allows us to capture mainly the quality and quantity of particle interactions with walls and obstacles, their response to a non-constant velocity profile of the fluid flow in the device and in case of dense suspension also the interaction among the particles. Proper modeling of cells rotation is crucial for long-term research of CTC adhesion to the surface of microfluidic devices.

To measure the rotation, we use the velocity component data in at the extremal points of the RBC surface. In this article we focus on one possible combination, which is obviously crucial and allows the analysis even in case of missing data in $z$ direction. If necessary, we prove the possibility to process other combinations of parameters in the same way.

Here, the extremal points were the minimum and maximum point of the cell surface in the $y$ direction ('right' and 'left' point) and we considered the $x$-component of velocity at these points. If these two values are equal, the interpretation is that the $\mathrm{RBC}$ does not rotate in the $x y$-plane. In the experimental data these velocities mostly differ. Their difference corresponds to the instantaneous rotation of the cell and the sign of the difference to the spin of rotation. Therefore, we have chosen the vector of differences of the $x$-components of velocities in $y$-extremal points as the basis for further statistical analysis. This vector is available for each of the $50 \mathrm{RBCs}$ and its length corresponds to the number of recorded values of cell motion.

The graphs of these differences (Figure 9) are very informative. One can observe significantly periodic behavior, surprisingly frequent changes in spin of rotation, as well as a large variation of this characteristic. This situation is very similar to the processing of vectors of absolute velocities of the RBCs in part 3.3, so we have also used similar methods for processing of gained data. 

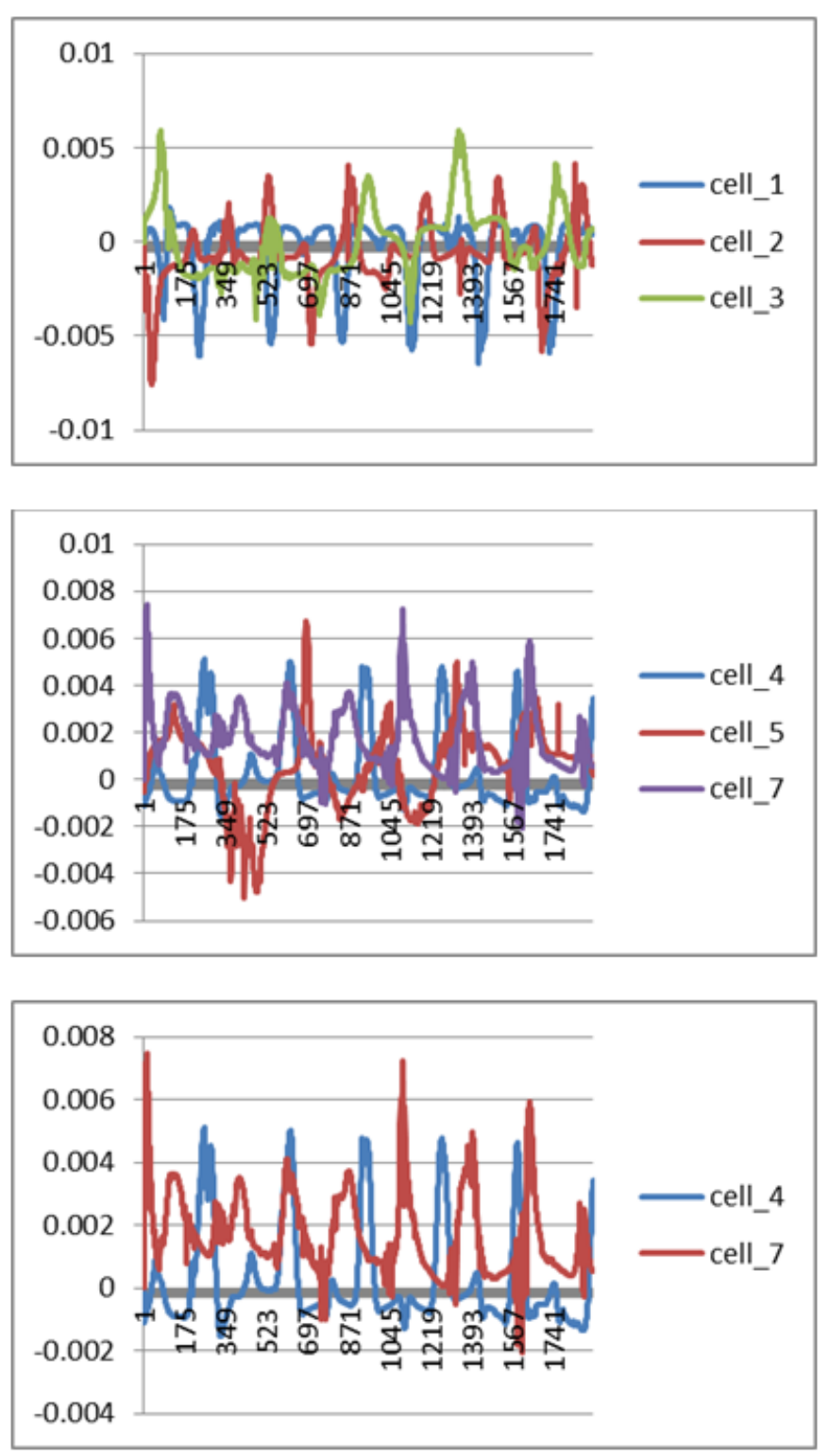

Fig. 9. Plots of rotation speed for several RBC's

First we have found the minimum and maximum value for each RBC (i.e. the maximum rotation in both spin directions), the average of the recorded values, the number of changes of spin and an integral (area between the curves of the $x$-component of the velocity at the $y$-extremal points). The latter value should correspond to the overall rate of rotation. (In fact, the integral was calculated as an integral sum of the absolute values of the tabulated velocity differences with width of 200 simulation steps and numericaly estimates this integral.)

The obtained vectors rot_min, rot_max, rot_avg, rot_switch and rot_integral with length 50 were independently sorted by the magnitude of their elements. These five vectors are the first rotation characteristic of the whole experiment. Their plots (Figure 10) confirm the expected (dis)similarities of experiments supported also by the KStest.
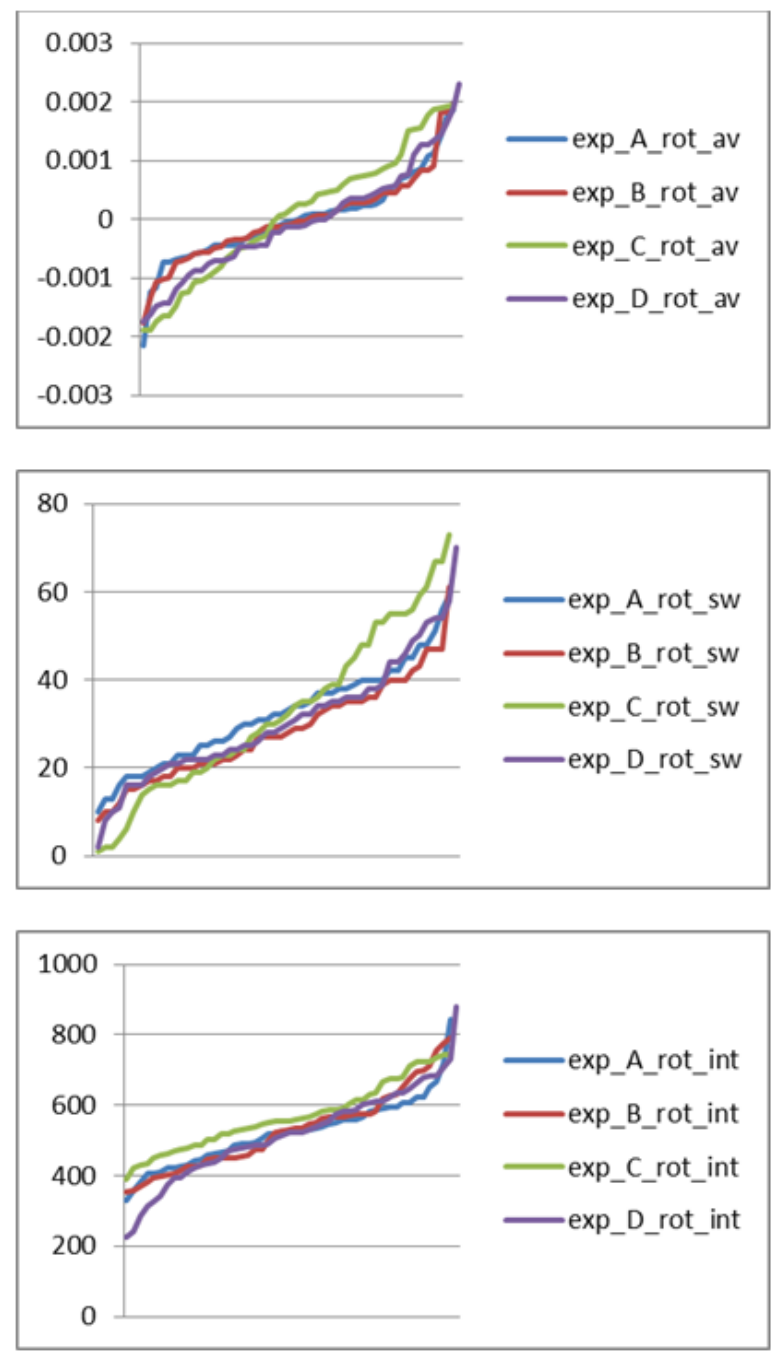

Fig. 10. Plots of rotational speeds, numbers of rotational shifts and integral for experiments $A, B, C$ and $D$ 
Another characteristic focused on periodic behavior of rotation velocity was investigated using DFT similarly as in the part 3.3. The results are shown in the following graphs (Figures 11 and 12) as frecvency vectors rot_freq_dom

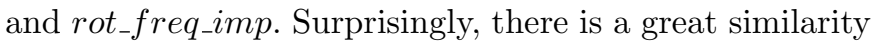
between the frequencies of velocity differences and absolute velocities, and as expected, the number of rotations changes rot_switch corresponds to double of frequencies rot_freq_dom values.
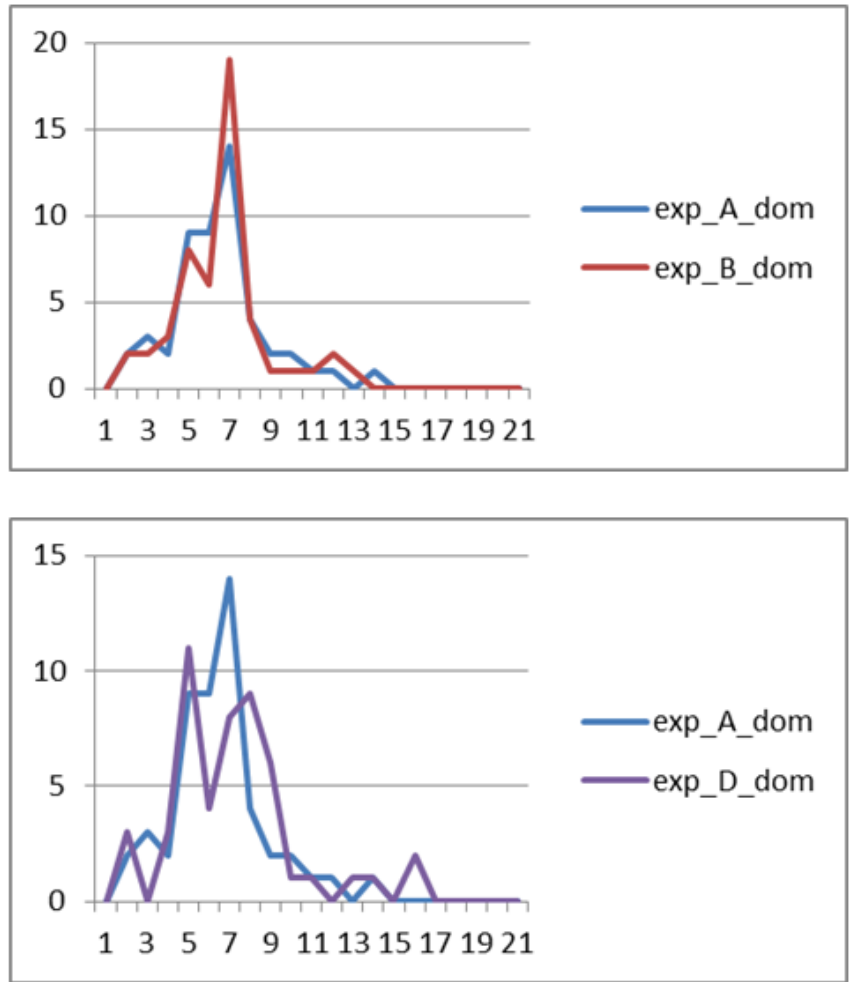

Fig. 11. Match of number of dominant frequencies for experiments $A$ and $B$ and their differencies for experiments $A$ and $D$
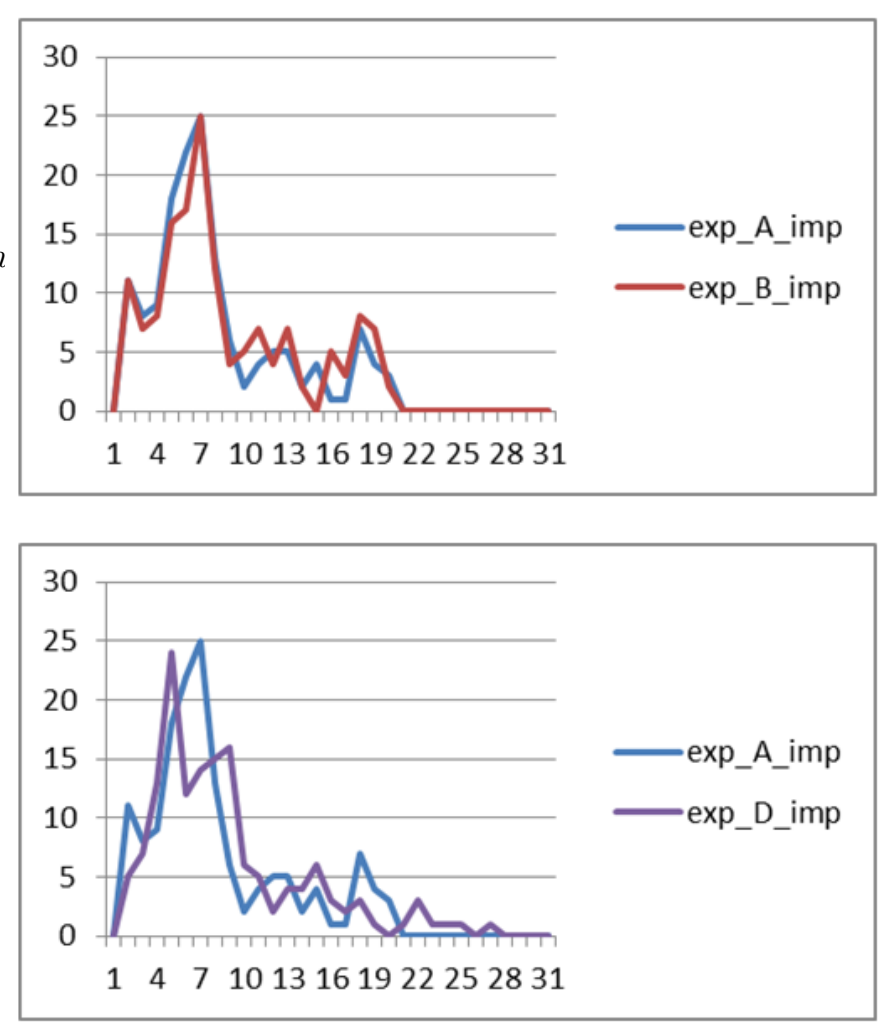

Fig. 12. Match of number of important frequencies for experiments $A$ and $B$ and their differences for experiments $A$ and $D$

The last graphs (Figure 13) show the results of a simple comparative experiment for some selected cells. If the idea of measuring the $\mathrm{RBC}$ rotation is correct, the differences in $x$-components of velocity in the $y$-extremal points should correspond to the differences in $y$-components of velocity in the $x$-extremal points ('first' and 'last' point of the cell surface). Pictured characteristics confirm the assumption.

Some differences arise just when part of the RBC is fixed or slowed down as a result of contact with an obstacle or with another object. These results are illustrated characteristics for three randomly selected RBCs. 

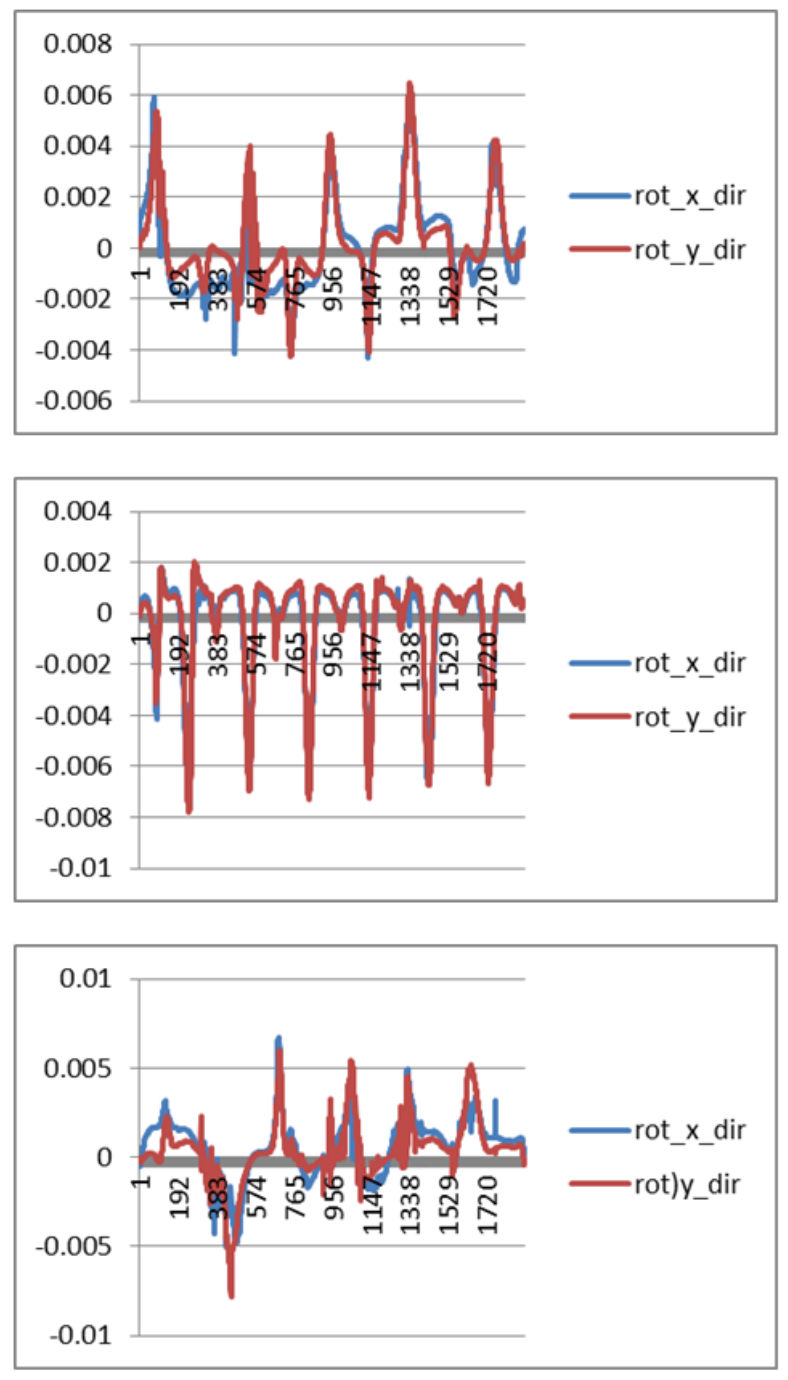

Fig. 13. Difference of rotational speed in $x$-axis and $y$-axis direction

\section{Snapshot Data Analysis}

As mentioned above, the data obtained from the snapshots are much simpler, static and provide fewer options for the characterization of cell motion during the simulation experiment. On the other hand, this kind of data is much easier to obtain using digital image processing of biological experiments. During our simulation experiments we acquire the snapshots after every 1000 simulation steps, but we have used only a small partion of them. For moni- toring the development and stabilization of the individual characteristics, we have used snapshots after every 100,000 steps, and after 300,000 steps, we have recorded the snapshot every 10,000 steps.

\subsection{Location of the cells in the device}

The first characteristic of the cell flow comes from data of the center position of individual RBCs (center of the circumscribed box with sides parallel to the axes). In this section we limit ourselves to analysis of their $x$-coordinates. If necessary, the method can be extended to the other coordinates. At the beginning of the simulation, the $x$-coordinates of RBCs range from $0 \mu \mathrm{m}$ to $100 \mu \mathrm{m}$. At the end of the simulation, after 381,000 simulation steps, depending on the starting position, trajectory and number of collision, the cells $x$-coordinates range between $352 \mu \mathrm{m}$ and $718 \mu \mathrm{m}$. The following figure (Figure 14) illustrates the developement of $x$-coordinates values for the whole RBCs 'bunch' during the simulation run.

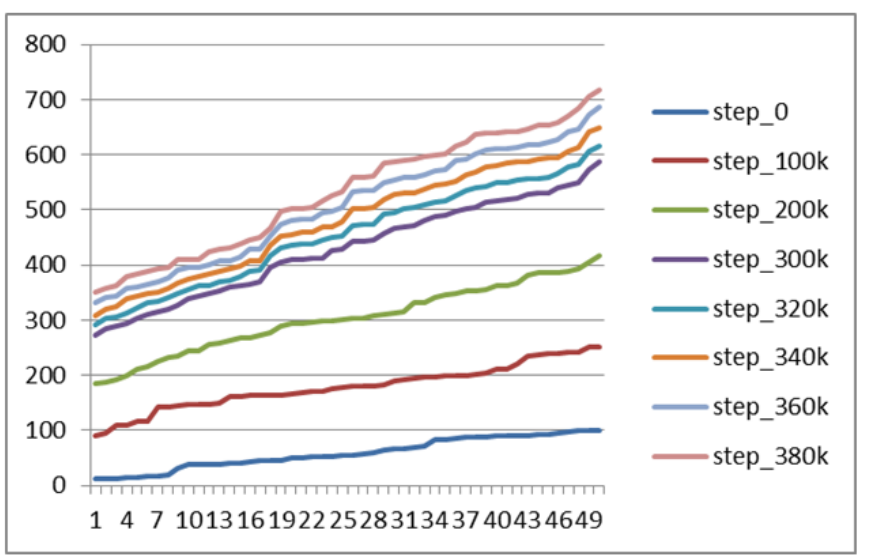

Fig. 14. Plot of $x$-coordinates of RBC's center for different simulation steps (in thousands) 
However, this characteristic is not our goal. In the biological experiments, we expect a much larger microfluidic device (with a periodic obstacle array) and the snapshot containing a large number of elastic objects continuosly filling interior of the device. Therefore, not the global RBC positions, but their positions in typical regions of obstacle array will by the important characteristic. Those would have to be defined specifically depending on the array type.

For our experiment, we have processed the RBCs $x$ coordinates in two steps. First, we replaced them by their remainder after devision by 100, which 'shifts' all coordinates to a common rectangle representing the basic periodic block of the channel. Then we divided them into three typical zones: entry zone which consists of two $10 \mu \mathrm{m}$ strips (15 to $25 \mu \mathrm{m}$ and 65 to $75 \mu \mathrm{m}$ ), through which the RBCs enter the narrow part in front of the obstacle belt. The exit zone consists of two $10 \mu \mathrm{m}$ strips (25 to $35 \mu \mathrm{m}$ and 75 to $85 \mu \mathrm{m}$ ), through which the cells leave these narrow parts. Third transit zone corresponds to the remaining 60 micrometers of the channel length without obstacles with a maximum flow space. The entry zone corresponds to the area with fast acceleration of the fluid flow, where also cell-cell and cell-wall collisions are the most frequent. Output zone is the area of cell flow deceleration, in which the collision situations are much less frequent. The transit zone correspond to the relatively steady state with a smooth flow of cells.

Our next proposed characteristic of the cell flow is the distribution of cells count in these zones. The first graph
(Figure 15) shows the time-evolution of this characteristic for the experiment $A$ using the snapshots after $300 \mathrm{k}, 320 \mathrm{k}$, 340k, 360k, 380k and 381k simulation steps. The distribution look quite stable, which confirms the suitability of this characteristic. The second graph compares these distributions for experiments $A, B, C, D$ after $381 \mathrm{k}$ simulation steps with high degree of similarity.
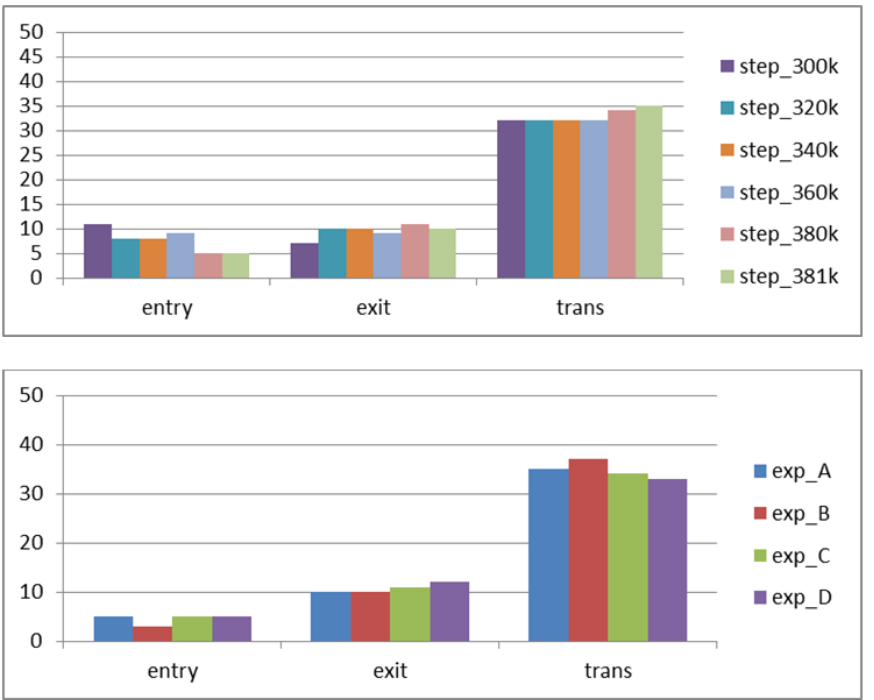

Fig. 15. Number of RBCs in zones for experiment $A$ during different time snapshots, comparison of numbers of cells in zones in simulation step $381 \mathrm{k}$ for experiments $A, B, C$ and $D$

A similar statistics can be created with lower respect to the specific shape of the experimental device. The following figures (Figure 16) show similar results for the distribution of cells count in sketchy created $10 \mu \mathrm{m}$ zones, covering whole length of basic chanel box. The values are not as stable as in the previous distribution, however, this may be due to the small number of RBCs in the experiment. We have performed a control experiment identical to the previous one but including only $20 \mathrm{RBCs}$. It turns 

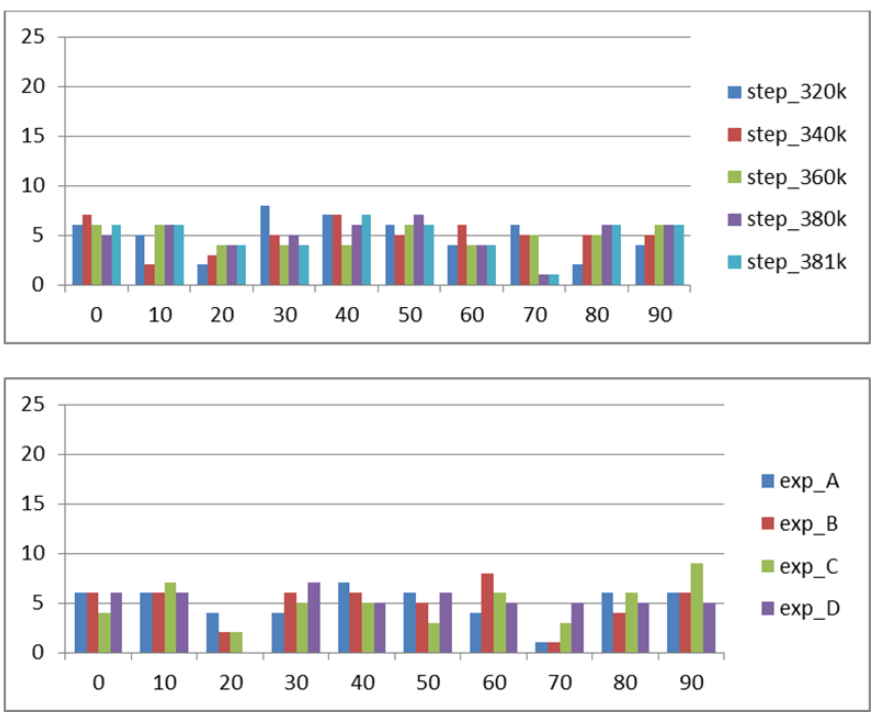

Fig. 16. Number of RBCs in $10 \mu \mathrm{m}$ bands for experiment $A$ during different time snapshots, comparison of numbers of cells in bands in simulation step $381 \mathrm{k}$ for experiments $A, B, C$ and $D$

out that the spatial distribution of cells has unsteady but periodic nature. This effect disappears when the number of cells in the model is increased to 50 .

For our type of channel topology and flow within the channel, the $x$-coordinates of the RBCs are crucial for description of the main directions of the cells movement. Another control experiment has shown that similar results may be obtained in an analogous analysis of the $y$-coordinates of cell positions.

\subsection{Cell skew in the device}

In section 3.4, we have described how important the investigation of the cell rotation characteristics is. The snapshots give us only limited options in this field, because they do not capture the dynamics of the simulations. Therefore, we have proposed a method for describing the RBC skew using the dimensions of a bounding box, defined as a cuboid containing the entire $\mathrm{RBC}$ and oriented in main axes direction, as mentioned in section 2.1. If the $\mathrm{RBC}$ is oriented by one way in the directions of the $x, y, z$ axes, the dimensions of the box are the same as the dimensions of the RBC, i.e. $7.82 \mu \mathrm{m} \times 7.82 \mu \mathrm{m} \times 2.56 \mu \mathrm{m}$. When the cell is skewed, the bounding box has different dimensions, which represent the rotational characteristics of the cell.

The obtained bounding box sizes were processed in two ways. For the simpler one, the values of the $x, y$ and $z$ dimensions (after 380k simulation steps) for all cells were independently sorted and stored in arrays $c u b \_x, c u b \_y$ and $c u b_{-} z$. These arrays form the first proposed characteristic. The graph (Figure 17) show a significant match of $c u b \_x$ vectors for experiments $A, B, C$ and $D$, but for the values of $c u b_{-} z$ vector some difference between the couple $A, B$ and couple $C, D$ of experiments arise.

It relates to interesting observation, that the distributions of $x, y$ and $z$ dimension of cuboids in a single simulation are not the same, i.e. the RBC skew is different for different axes. Comparing the graphs for simulation $A$ and $D$ (Figure 18) shows, then the differences between $c u b \_x, c u b_{-} y$ and $c u b_{-} z$ distribution (mind the switch of $c u b_{-} y$ and $c u b_{-} z$ graphs position) can characterize the experiment too.

The second skew characteristic measures nearly standardly the normalized square differences of the current bounding box dimensions and dimensions of the bounding box of RBC aligned with the coordinate axes. The small difference consist in fact, that the values of the box 

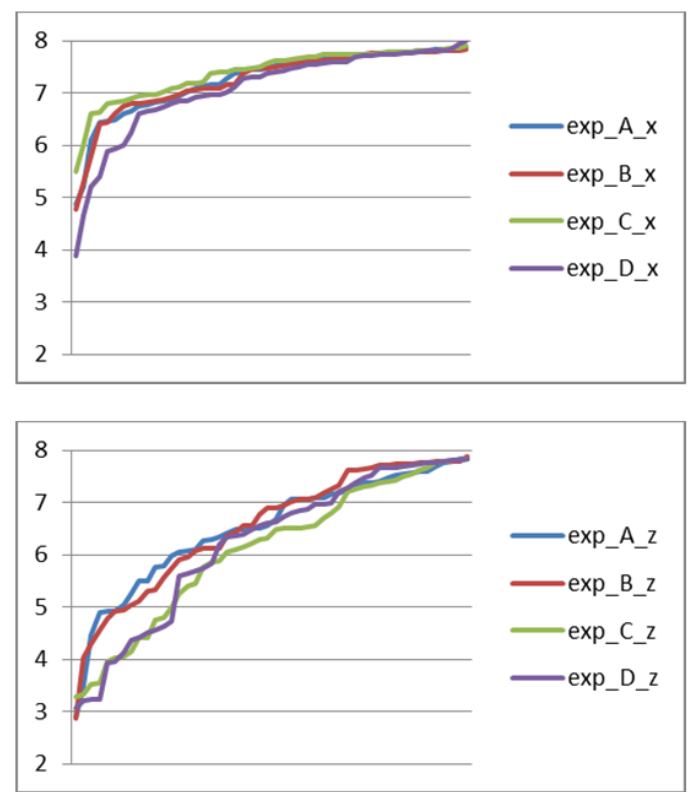

Fig. 17. Plots of $x$-sizes and $z$-sizes of cuboids for experiments $A, B, C$ and $D$
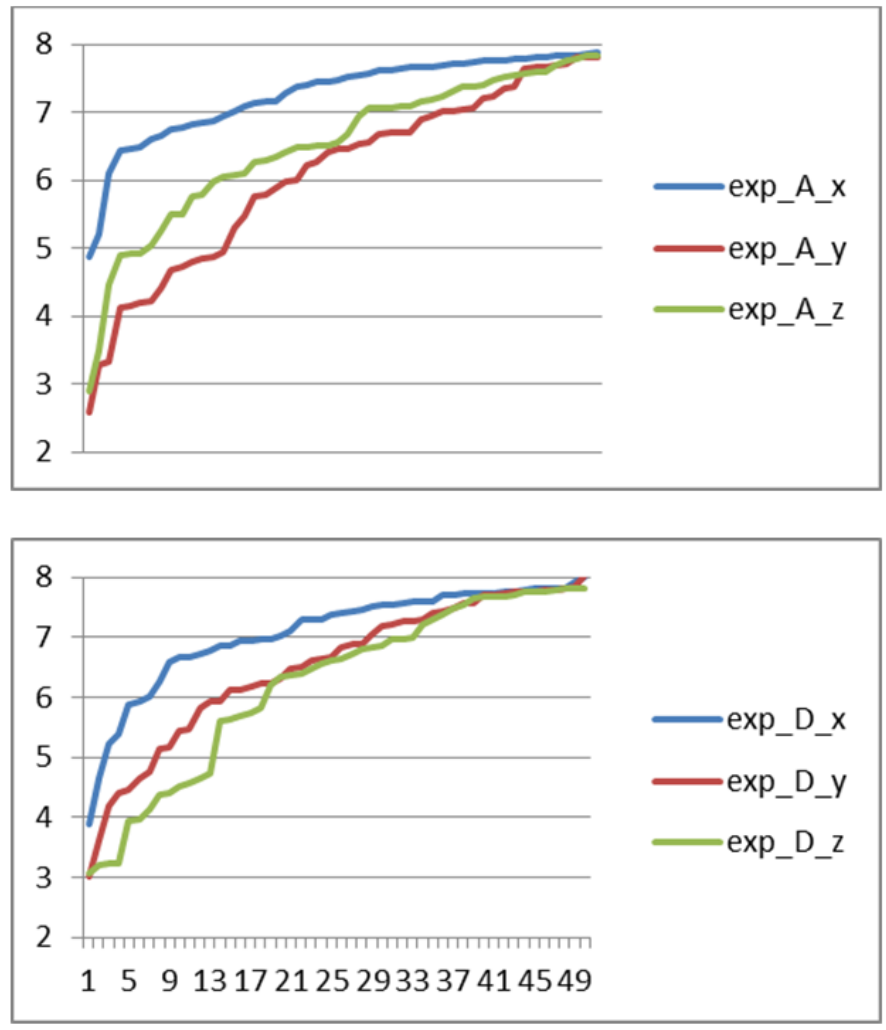

Fig. 18. Comparison of $x, y$ and $z$-sizes of cuboids for experiments $A$ and $D$ dimensions are first sorted by size. Than the two largest values are compared to the diameter of $\operatorname{RBC}(7.82 \mu \mathrm{m})$ and the smallest one is compared to the thickness of the RBC $(2.56 \mu \mathrm{m})$. In this way, in order to simplify, we intentionally decrease mentioned difference of the dimension in the direction of the individual axes. Nevertheless, the acquired characteristic still has very good properties. The measured values are stored in a vector cub_sq_err. After being sorted by size, this vector represents another skew characteristic of the RBC in the snapshots of simulation experiments.
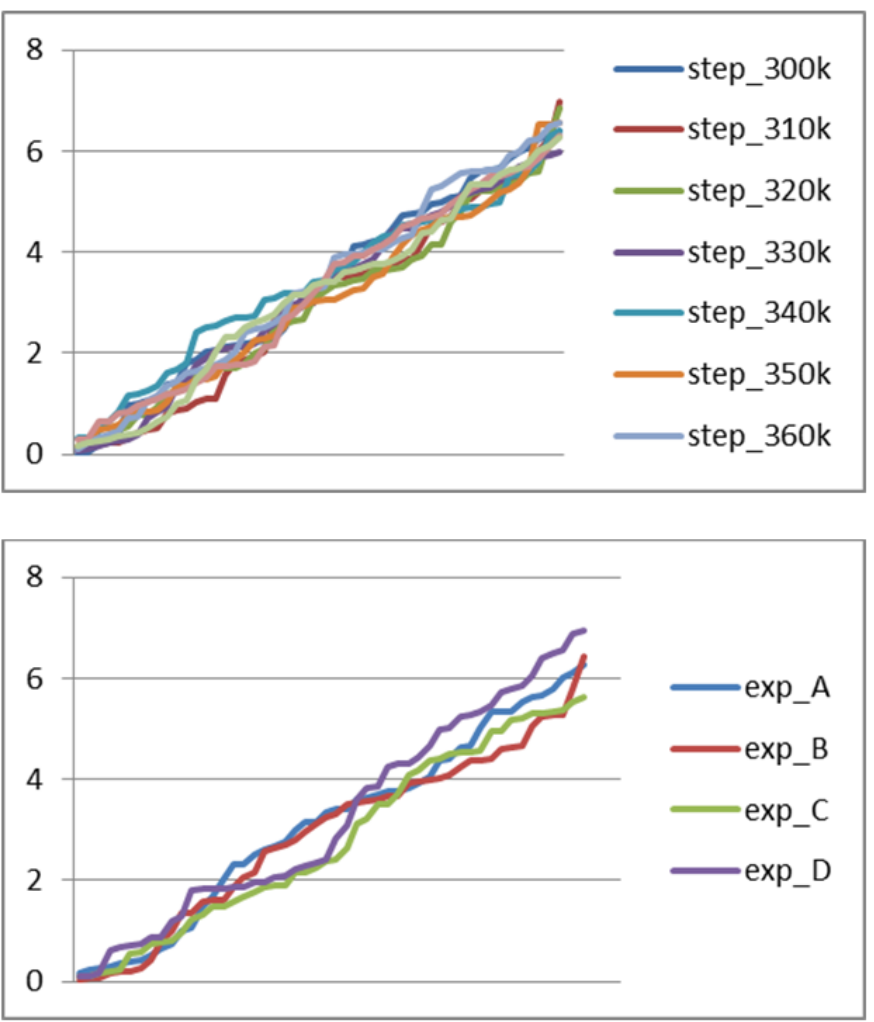

Fig. 19. Stability of characteristic for all snapshots from experiment $A$, comparison of square errors of cuboid rotation for experiments $A, B, C$ and $D$ 
The first graph (Figure 19) illustrates the stability of the vector in snapshots between 300,000 and 380,000 simulation steps for simulation $A$. The second graph shows a comparison for the snapshots of experiments $A, B, C$ and $D$ after the 380,000 simulation steps.

\section{Used technologies}

For the simulation experiments, we have used the version 3.2.0 of computational package Esspresso, especially its OIF framework. The generated data files were subsequently processed by algorithms developed in

C\#inVisualStudio2010 into the data files suitable for presentation of results and further statistical processing.

The statistical processing was done in Matlab, version 7.11.0.

The following simulation parameters were used:

fluidfriction_coef $f=0.025865531914893616$,

fluid_density $=1.0$, fluid_viscosity $=1.5$,

fluid_force $=0.0005$, timestep $=0.2$ microseconds,

lb_agrid $=1.0$, number_simulation_steps $=381,000$.

The RBC was modeled with standard triangulation with

141 nodes and with its external dimensions of

$7.82 \mu \mathrm{m} \times 7.82 \mu \mathrm{m} \times 2.56 \mu \mathrm{m}$.

For the elastic modulus of RBC, the used coefficients were $k s=0.0044, k b=0.0715, k a l=0.005, k a g=1, k v=$ 1.25 .

\section{Conclusions}

The proposal of statistical methods for mutual comparison of conformity or similarity within the simulations and real experiments, with the flow of a large number of objects, are in our opinion a relatively new area of research. The characteristics suggested in the article represent our first attempt at defining and processing methodology. The presented results indicate their stability and satisfactory resolution of the proposed methods. They become a good starting point for further development of the topic. It should focus on the following areas:

- More detailed verification of stability of characteristics and verification of distinguishing capabilities in a case of a fine changes of the parameters of the experiment,

- Collection of comparable data from real experiments and simulations of other simulation tools, and comparing the characteristics.

- Further development and widespread use of characteristics.

- Extending the use of the characteristics to measure the quality of simulations in terms of optimizing the properties of microfluidic devices.

\section{Acknowledgements}

The authors acknowledge the experimental data from Dr. Giulia Mazza, Center for Integrated Sensor Systems, Danube University Krems, Austria. Her work is supported by the NÖ Forschungs- und Bildungsges.m.b.H. (NFB) through the Life Science Calls (Project ID: LSC13-024). 


\section{References}

1. K. Bachratá and H. Bachratý, On modeling blood flow in microfluidic devices, ELEKTRO 2014: 10th International Conference, IEEE, ISBN 978-4799-3720-2, 2014, pp. 518-521

2. I. Cimrák, K. Bachratá, H. Bachratý, I. Jančigová, R. Tóthová, M. Bušík, M. Slavík and M. Gusenbauer, Objectin-fluid framework in modeling of blood flow in microfluidic channels, Comunications, Scientific Letters of the University of Zilina, vol. 18/1a, 2016, pp. 13-20

3. M. Bušík, I. Jančigová, R. Tóthová and I. Cimrák, Simulation study of rare cell trajectories and capture rate in periodic obstacle arrays, Journal of Computational Science, 2016

4. X. Li, Z. Peng, H. Lei, M. Dao and G. E. Karniadakis, Probing red blood cell mechanics, rheology and dynamics with a two-component multi-scale model, Philosophical Transactions Series A, Mathematical, Physical and Engineering Sciences, 2014

5. D. A. Fedosov, J. Fornleitner, J. L. McWhirter, K. Müller, H. Noguchi, M. Peltomäki and G. Gompper, Blood Flow in silico: From Single Cells to Blood Rheology, 4th Micro and Nano Flows Conference UCL, 2014

6. R. Tóthová, I. Jančigová and M. Bušík, Calibration of elastic coefficients for spring-network model of red blood cell, International Conference on Information and Digital Technologies (IDT), 2015, pp. 376-380

7. I. Cimrák, M. Gusenbauer and I. Jančigová, An ESPResSo implementation of elastic objects immersed in a fluid, Computer Physics Communications, vol. 185, 2014, pp. 900-907

8. H. Basu, A. K. Dharmadhikari, J. A. Dharmadhikari, S. Sharma and D. Mathur, Tank treading of optically trapped red blood cells in shear flow, Biophysical Journal, vol. 101, 2011, pp. 1604-1612

9. K. Tsubota and S. Wada, Elastic force of red blood cell membrane during tank-treading motion: Consideration of the membrane's natural state, International Journal of Mechanical Sciences, vol. 52, 2010, pp. 356-364 